\title{
How To, or Not To Intervene - Some Ethical Dilemmas Encountered by Mental Health Professionals
}

\author{
Supreet Kaur Bhasin ${ }^{1}$, Aditi Sharma ${ }^{2}$, Triptish Bhatia ${ }^{3}$, Smita N. Deshpande ${ }^{4}$ \\ ${ }^{1}$ University of Delhi \\ ${ }^{2}$ Developmental Psychologist, Children First, Safdarjung Development Area, New Delhi \\ ${ }^{3}$ Research Unit, Centre of Excellence in Mental Health, PGIMER-Dr. RML Hospital, New Delhi \\ ${ }^{4}$ Professor \& Senior Consultant, Centre of Excellence in Mental Health, PGIMER-Dr. RML Hospital, \\ New Delhi \\ Corresponding Author: Smita Deshpande \\ E-mail: smitadeshp@gmail.com
}

\begin{abstract}
Background: Indian mental health care professionals share some ethical concerns similar to those of their Western counterparts e.g. boundary violations, confidentiality issues, caregiving by multiple healthcare workers of varying competence, as well as additional issues around decisional autonomy, cultural issues around psychometric assessments and chiefly, hierarchical relationships with healthcare workers.

Methods: Authors (SKB, AS) shadowed young healthcare professionals across duties such as ward rounds, detailed history taking, while conducting psychometric assessments and during psychotherapy sessions but did not directly interact with the patients. The epistemological position of authors was social constructionism, with the method of enquiry being participant as observer, employing unstructured, direct observation.

Results: A total of 11 post graduate trainees ( 7 in clinical psychology, 4 in psychiatry) and 2 certified junior clinical psychologists were observed for a period of 60 days. Key themes emphasizing the ethical dilemmas encountered by mental health care professionals were extracted from case notes.

Discussion: Major themes found were: confidentiality vs clinical demands and constraints, autonomy vs. consent and assent, informed consent vs. deception, clinical judgement vs. results on standardized assessment instruments and power hierarchy vs equivalence via disclosure.

Conclusion: The current exploratory study provides a small but significant insight into the challenges and ethical dilemmas encountered by young mental health professionals. It highlights the complex problem of abiding by the ethical principles, growing need to make culture specific ethical decisions for an acceptable model of mental health treatment and developing a healthy trusting relationship between healthcare providers and patients, and their families. Students and faculty need to be alert to ethical issues, teaching of ethical principles following the case-based approach advocated by the UNESCO Bioethics Chair may be the best option. Role plays and other active modes of learning need to be started as soon as students begin interacting with patients.
\end{abstract}

Keywords: boundary violations, confidentiality, constraints, ethics, dilemmas, mental health. 


\section{INTRODUCTION}

Ethical dilemmas and concerns are an important part of any healthcare system, but ethical treatment needs to be tailored to local realities and constraints [1-2]. Indian mental health care professionals share some ethical concerns similar to those of their Western counterparts [3] such as boundary violations, confidentiality issues, caregiving by multiple healthcare workers of varying competence, however, they also face additional challenges like- issues around decisional autonomy, cultural issues around psychometric assessments and chiefly, hierarchical relationships with healthcare workers [4-8].

Ethical dilemmas and concerns are thus a part and parcel of the Indian medical system. For instance, confidentiality has to be often compromised, primarily due to the lack of proper infrastructure [9]. Similarly, both psychiatrists and clinical psychologists are aware of but do not report non-sexual boundary violations. These include receiving gifts, making friends, accepting free waivers and services from patients [10]. The plausible reasons for toleration of these boundary violations are that such behaviors are believed to be culturally sanctioned [10], out of fear of making the patient feel rejected [7] as well as lack of appropriate guidelines defining the professional boundaries of a doctor patient relationship in keeping with our social milieu [11]. Therapists in addition face peculiar dilemmas when faced with patients whose belief systems, values and moral principles are incongruent with their own: around marriage, gender identity, abusive violent interpersonal relationships or extramarital relationships [7,12]. Likewise, certain psychotherapeutic principles may not resonate with therapist or patient, and their regular clinical use risks wounding the mind, spirit and body of the patients [13-14]. In such cases, the quality of the relationship and treatment by the therapist is likely to be affected, yet in India, a referral might not always be possible due to paucity of available resources [9].

An Indian mental health care worker hence, is swamped with ethical dilemmas, but literature specifically focusing on the forms and nature of these challenges as well as on the means of resolving them is less common [15]. Furthermore, there are no culture sensitive ethical guidelines for psychiatric and psychological health treatment in India $[2,8]$.

Emerging out of these concerns, the objective of the current study was to develop an understanding of some ethical challenges and dilemmas observed by the authors in a free, tertiary care, post graduate teaching government hospital in New Delhi. This cross-sectional qualitative study used field notes of unstructured observation as the research tool. Unstructured observation was used because it accounts for the context, is sensitive to the impact of physical environment, provides comprehensive understanding of the varied interactions and can be holistic in nature [16]. Field notes were descriptive (sensitive to the factuality, physical settings, context etc.) as well as reflective (indicative of unanswered questions, thoughts and critical thinking of the researcher). Field notes were then re-examined and analyzed to form themes.

\section{METHODOLOGY}

Detailed observations were carried out by the first two authors at the Department of Psychiatry and Clinical Psychology at the Dr. Ram Manohar Lohia Hospital in New Delhi as a part of their post graduate internship training. Authors were expected to shadow clinicians as they went about their regular work. Field notes were maintained regularly and discussed. The authors (SKB and AS) shadowed the mental healthcare trainees and professionals but did not directly interact with the patients. Patient encounters were observed both when the patient (and caregiver) presented to the OPD for the first time, as well as during follow ups. All behavior occurring during the patientdoctor interaction were noted, without any pre-decided categories. The epistemological position of the authors was social constructionism, with the method of enquiry being participant as observer, employing unstructured, direct observation. 


\section{RESULTS}

A total of 11 trainees ( 7 post graduate non-medical trainees in clinical psychology, 4 post graduate medical trainees in psychiatry) and 2 certified junior clinical psychologists were observed for a period of 60 days (510 hours) from 26th May 2016 - 22nd July 2016. These 13 mental health professionals were observed during ward rounds, detailed history taking, while conducting psychometric assessments and during psychotherapy sessions. However, no senior clinicians or psychology faculty were observed. Key themes with regard to the ethical dilemmas encountered by mental health care professionals were extracted from the field notes, using the word 'patient' when medical interventions are described, and 'clients' when psychological tests or interventions are described.

Clinical constraints and demands vs Maintaining Confidentiality: Being a government hospital, the OPD was usually crowded with huge number of patients, with scarce resources. Consequently, young junior doctors and psychologists found themselves sitting in cramped spaces, several of them huddled together, each attending to a patient and their primary caregivers in one room. Working under such circumstances, made it impossible to observe confidentiality, and overhearing of personal narratives and challenges of the patients was common. Contrarily, some people found solace in being stuck in similar compromised situation of healthcare as well as of mental illnesses. Autonomy vs Absence of Assent/consent: Children may be reluctant to participate in psychological assessments, mandated for assessing their IQ, learning disabilities or behavioral problems, but they had to obey their parents. Assent from children was not mandated for psychometry or psychotherapy.

Informed consent vs Use of Deception: Persons presenting to the department were often cognitively impaired. Occasionally, 'deception' was employed albeit with the intention of helping the patient/caregivers: using placebos for drug dependent patients to ensure abstinence, or agreeing with the patient's cognitive bias to obtain full details of his or her thought disorder (could the validation further decrease insight or prolong the course of treatment?), using a substitute term (perhaps less serious or less stigmatizing) instead of naming the actual diagnosis. During management, practices observed were: offering or using only one form of psychotherapy without considering client conviction, convenience and other factors such as the client-therapist interaction and the context. In the main, these practices were observed without malicious intent and although not deception in the true sense, did limit choices.

Clinical Judgment vs Standardized assessment instruments: Sometimes, there was discrepancy in clinical judgment about the client's functionality and the score obtained on psychometric assessment tool. For instance, individuals with substance abuse feigning a lower score on dependency index, while their personal narratives and family's description conveyed otherwise. Similarly, young children performed poorly on the psychological tests of learning disability and IQ, but their functionality appeared otherwise.

Power Hierarchy vs Equivalence via Disclosure: It was not uncommon to find the clients perceiving the therapists as their guide and an all-encompassing source of knowledge. Clients did not wish to enter the therapeutic alliance as equals, and instead desired to be led by the insights of the therapist and to surrender to him/her. Despite being cognizant about the advantages of selfdisclosure, during the observation not a single episode of self-disclosure was observed.

\section{DISCUSSION}

While many themes highlighted in the results above are commonly practiced by healthcare workers not only in Psychiatry, Psychology but also in many other fields of medicine (for instance, life threatening situations, incurable diseases), these issues need to be described and debated.

The inability to provide full privacy because of infrastructural limitations or intrusion by family caregivers was common. Confidentiality, by ensuring a trusting relationship between the doctor and the patient/client, encourages treatment adherence and fosters a trusting relationship. Public healthcare systems are unable to provide for the needs of the burgeoning urban population, due to overcrowding and old buildings, not equipped for the huge inflow [9]. However, in the socio- 
cultural environment of a country like India, privacy means being barred from an inter-dependent and close-knit society [17]. Therefore, the ability of the patients/clients and their caregivers to be unguarded about their vulnerable mental health status, generated a feeling of togetherness.

Most family members assumed that, since they were bringing the patient to the hospital, and would manage the patients at home, they deserved to be fully informed. Additionally, in case of patient with psychotic symptoms, and resulting conflicts, the family pressurized the therapist to divulge confidential information, which is in congruence with previous reports $[4,5,18]$.

Family members felt even more offended in cases where their child perceived the client-therapist relationship to be more trustworthy. The prevailing social belief is that children should not hide anything from the family. Hence, parents felt justified in demanding all information about the child [18-19]. In India, due to the vertical collectivistic culture between the elderly and children in family, the power of parents in decision making is near absolute $[22,6]$. The authoritarianism of Indian parents reduced the autonomy of young children and adolescents, so the therapists had to overlook the child's assent and prioritized parents' consent. In case of children and adolescents, assent should be sought before performing any psychological assessments [20]. Children, by the age of 9 years possess the cognitive capacity to decide about participating in an activity [21]. Assent was seldom sought in the hospital setup, and where the child and parents disagreed about participating in the psychiatric or psychological treatment, the decision of the parents was abided by, perhaps because parents sought help for disability certification which would materially benefit both the child and the family [23].

In the majority of instances, parents accompanied their adolescents to therapy sessions. Parents felt that their child lacked capacity to express his/her own wishes due to inhibitions, or because they believed that owing to the psychiatric illness, their child did not know his/her best interest. Therefore, the parents insisted that they be included in all the clinical processes, as also described by others $[15,18]$. Yet, adhering to parental expectations was problematic for the therapist in case of adolescents, who usually insisted on being treated as an autonomous individuals and perceived therapy as safe space for expressing individuality.

Informed consent was compromised upon not only for children and adolescents, but sometimes also for adults who were believed to be incompetent. Sometimes, the family believed that in case the patient got the liberty to choose the treatment, s/he would deny the problem, which would worsen her/his physical and psychiatric health.

Deception has been defined as an act, wherein the investigator either provides false information so as to misinform the patient or withhold true information from the patient [24]. Interviewers used deception to gather detailed symptomatology from challenging patients by validating their point of view-e.g. by agreeing to their versions of delusions or hallucinations. The motive of doing so was to benefit the patient, yet it was also a form of misinformation. Using a substitute term for the actual diagnosis was another form of deception. The efficacy of a therapy may depend on patient conviction, convenience and other factors such as the patient-therapist interaction and the context instead of the disease; asserting supremacy of one form of therapy over other could be questionable $[25,5]$, yet clients were not offered a choice. Firm belief in and insistence upon medications even in case of mild anxiety and depression, despite evidence to the contrary [26] was widespread.

If clinical judgement was inconsistent with psychometric results, psychology trainees questioned their own competency, or feared introducing subjective bias in empirical testing despite evidence to the contrary [27, 28]. Consequently, despite feeling unconvinced with the results of psychometric testing, the psychology trainees wondered whether to abide by the unconvincing psychometric score or their own clinical judgment.

Historically, only the sacred healers were thought to possess ultimate knowledge of medicine and life [29]. Consequently, the clients extended similar beliefs and emotions towards the contemporary healers- the health professionals, exhibited similar relationship dynamics in professional relationships $[5,15]$. Perhaps, the clients trusted their therapists and held strong blind faith unto them $[4,7]$. However, living up to the ideal image of a healthcare professional impinged upon other inter-related ethical principles such as self-disclosure. Self-disclosure by the therapist positively affect the therapeutic alliance, since it reduces the taboo felt by the patient. Self- 
disclosure is a key element in creating an empathetic bridge between the professional and the patient, and more importantly, it being insightful for the therapist, leading to fresher perspectives to therapy [30]. But in the present situation, personal revealing statements were viewed as boundary violation and thought to diminish the trustworthiness towards treatment. This often created an ethical conflict within the therapists, since on one hand, they were withholding from self-disclosure despite knowing its benefits, but on the other were equally preventing harm to the patient by not risking their loss of faith in the treatment. Therapists assumed that self-disclosure would invariably translate into developing multiple relationships with the patient, especially in the case of children and adolescents, and choosing not to self-disclose protected them from entering into multiple relationships like friendship or career mentorship with the children. Although multiplicity of relationships could mean boundary violation on part of the therapist and breach of ethical principles yet refraining from self-disclosure also impacted the inspiration and direction the children expected to find as part of the psychological contract in the embodied 'guru-chela' [31] relationship.

It was not uncommon for patients to visit their consultants and therapists with gifts or sweets, as a token of gratitude. Did the cultural sanctioning of souvenirs or gifts as gratitude overlook the boundary violation taking place? Despite awareness about the non-sexual and sexual boundary violations, medical healthcare professionals in India are said to be underskilled - even incompetentin establishing healthy boundary norms between themselves and the patient [4,32]. This may be due to ambiguous limitations of self-disclosure. Consequently, their attitude towards selfdisclosure oscillated between polite discouragement, varying from self-protecting self-disclosure, to undue disclosure ensuing a role reversal between the doctor and patient [33].

In addition, the healthcare professionals, sometimes also chose to use less-than-clear explanations to disclose the diagnosis so as to soften its impact. This helped in maintaining a trusting and safe relationship between the therapist and client, presumably ensuring compliance to treatment and family acceptance. This is in congruence with several reports that health care professionals exercise deception to manage the behavior of the patient [34] and to manage the patient's environment [35].

Our study thus sheds light on the multi-layered, culture specific ethical dilemmas faced by mental health professionals, but is limited by small sample size, restriction to single medical institution and lack of triangulation with observation. Moreover, no senior clinicians or clinical psychologists were observed. Observations were part of an internship by the first two authors; hence we may not have captured the nature of all forms of ethical quandaries or the mechanisms of resolving them.

\section{CONCLUSION}

The current study provides a small but significant insight into the challenges and ethical dilemmas encountered by young mental health professionals on an everyday basis. This exploratory study points at the complex problem of abiding by the ethical principles, growing need to make culture specific ethical decisions and guidelines for an acceptable model of mental health treatment, and developing a healthy trusting relationship between healthcare providers, patients, and their families. Students and faculty need to be alert to ethical issues, teaching of ethical principles following the case-based approach advocated by the UNESCO Bioethics Chair may be the best option. Role plays and other active modes of learning need to be started as soon as students begin interacting with patients.

\section{REFERENCES}

1. Hays PA. Integrating evidence-based practice, cognitive-behavior therapy, and multicultural therapy: Ten steps for culturally competent practice. Profess Psychol Res Pract 2009;40(4):354-60.

2. Asnaani A, Hoffmann S. Collaboration in Culturally Responsive Therapy: Establishing a Strong Therapeutic Alliance across cultural lines. J Clin Psychol 2012;6:187-97.

3. Pope KS, Vetter VA. Ethical dilemmas encountered by members of the American Psychological Association: A national survey. Amer Psychol 1992;47(3):397-411.

4. Isaac R. Ethics in the practice of Clinical Psychology. Indian J Med Ethics 2009;6(2): 69-74. 
5. Manickam, LSS. Psychotherapy in India. Indian J Psychiatry 2010;52(Suppl 1):S366-70.

6. Chadda RK, Deb KS. Indian family systems, collectivistic society and psychotherapy. Indian J Psychiatry 2013;55:299-309.

7. Bhola P, Sinha A, Sonkar S, Raguram A. Ethical dilemmas experienced by clinical psychology trainee therapists. Indian J Med Ethics 2015;12(4):206-12.

8. Konar D. Why Psychotherapy never picked up in India: Could there be a solution? Eastern J Psychiatry 2015;18(1): 39-41.

9. Bajpai V. The Challenges Confronting Public Hospitals in India, their origins and possible solutions. Adv Health, 2014;1-27.

10. Kurpad S, Machado T, Galgali R. Is there an elephant in the room? Boundary violations in the doctor-patient relationship in India. Indian J Med Ethics 2010;7(2):76-81.

11. Lehavot K, Barnett JE, Powers D. Psychotherapy, professional relationships, and ethical considerations in the MySpace generation. Profess Psychol Res Pract 2010;41:160-6.

12. Elliot GR. When values and ethics conflict: The counsellor's role and responsibility. Alabama Counseling Association Journal 2011;37(1):39-45.

13. Sindhu G. The Application of western Models of Psychotherapy by Indian Psychotherapists in India: A Grounded Theory. Antioch: Antioch University Repository and Archive, 2017.

14. Christopher JC, Wendt DC, Marecek J, Goodman DM. Critical Cultural Awareness: Contribituions to a globalizing psychology. Amer Psychol 2014;69(7):645-55.

15. Agarwal AK. A review of Indian psychiatry research and ethics. Indian J Psychiatry 2010;52(Suppl 1):S297-305.

16. Silverman D. Interpreting Qualitative Data: Methods for Analysing talk, text and Interaction. 1st ed. London: Sage Publications; 1993.

17. Neki JS. Psychotherapy in India. Indian J Psychiatry 1977;19:1-10.

18. DeSousa A. Ethical issues in child and adolescent psychotherapy: a clinical review. Indian J Med Ethics 2010;7(3):157-61.

19. Ascherman LI, Rubin S. Current ethical issues in child and adolescent psychotherapy. Child Adolesc Psychiatr Clin North Amer 2008;7(1):21-35.

20. Indian Association of Clinical Psychologists. Code of Conduct. (Adopted by IACP, circulated to its members). Lucknow: IACP Secretariat; 1993; 2014.

21. Leiken S. Minors assent, consent, or dissent to medical research. IRB Review on Human Subject Research 1993;15:1-7.

22. Malhotra S, Subodh BN. Informed Consent and ethical issues in Pediatric psychopharmacology. Indian J Med Research 2009;29(1):19-32.

23. Paraspoor A., Paraspoor MD, Rezai N, Asgari F. Autonomy of children and adolescents in consent to treatment: Ethical, Jurisprudential and Legal Considerations. Iran J Pediatr 2014;2:241-8.

24. Wendler D, Miller F. Deception in the Pursuit of Science. Arch Intern Med 2004;164:597-600.

25. Shamasundar C. Some personal reflections relating to psychotherapy. Indian J Psychiatry 2008;50(3):301-4.

26. Bernstein M, Brown W. The placebo effect in Psychiatric practice. Curr Psychiatry 2017;16(11):2934.

27. Grove WM, Zald DH, Lebow BS, Snits BE, Nelson CE. Clinical vs. mechanical prediction: A metaanalysis. Psychol Assess 2000;12:19-30.

28. Sanders S, Doust J, Glasziou P. A systematic review of studies comparing diagnostic clinical prediction rules with clinical judgment. PloS One 2015;10(6).

29. Saini A. Physicians of ancient India. J Family Med Prim Care 2016;5(2):254-8.

30. Audet C. Client perspectives of therapist self-disclosure: Violating boundaries or removing barriers? Counsel Psychol Quart 2011;24(2):85-100.

31. Neki JS. Guru-Chela relationship: The possibility of therapeutic paradigm. Am J Orthopsychiatry 1973;43:755-66.

32. Khan M. Boundary Violations and The Curtain of Culture. Indian J Med Ethics 2010;6:76-81.

33. Beach MC, Roter D, Larson S, Levinson W, Ford DE, Frankel R. What do physicians tell patients about themselves? A qualitative analysis of physician self-disclosure J Gen Intern Med 2004;19:911-6.

34. Tuckett AG. The experience of lying in dementia care: a qualitative study. Nurs Ethics 2012;19(1):7-20.

35. Seaman AT, Stone AM. Little White Lies: Interrogating the (Un)acceptability of Deception in the context of dementia. Qual Health Res 2017;2:60-73. 
Acknowledgements: The salary of Dr. Triptish Bhatia is supported by the Stanley Medical Research Institute (07R-1712 to VLN) and National Coordinating Unit ICMR for NHMP Projects funded by Indian Council of Medical Research. The content of this paper is solely the responsibility of the authors and does not necessarily represent the official views of the funding agencies. We gratefully acknowledge the leadership of the movement for global bioethics- UNESCO Bioethics Haifa, and their leadership to organize the World Bioethics Day on October 19. This paper is an extension of the prize -winning poster on WBD 2019.

Financial grants for this paper: Nil

Conflict of interest: None 